\title{
Detection and Analysis of Skin Cancer in Skin Lesions
}

\author{
Amruta M. Gajbar ${ }^{1}$, A.S.Deshpande ${ }^{2}$ \\ $\operatorname{ME}\left(2^{\text {nd }}\right.$ Year)Student, Department of Electronics and Telecommunication Engineering \\ (Signal processing) JSPM Wagholi, Pune, Maharashtra, India ${ }^{1}$ \\ Assistant Professor, Department of Electronics and Telecommunication Engineering \\ (Signal processing) JSPM Wagholi, Pune, Maharashtra, India ${ }^{2}$
}

\begin{abstract}
During the last years, computer-vision-based identification systems are employed in many hospitals and medical specialty clinics, aiming largely at the first detection of carcinoma, and additional specifically, the popularity of melanoma neoplasm. In this paper, we tend to review the state of the art in such systems by initial presenting the installation, the visual options used for skin lesion classification, and therefore the strategies for outlining them. Then, we have a tendency to describe a way to extract these options through digital image process ways, i.e., segmentation, border detection, and color and texture process, and that we gift the foremost outstanding techniques for skin lesion classification. Image segmentation is vital|a crucial|a vital|a very important $\}$ task in analysing dermoscopy pictures because the extraction of the borders of skin lesions provides important clues for correct designation. In this paper, we have a tendency to introduce a replacement mean shift based mostly fuzzy c-means formula that needs less machine time than previous techniques whereas providing smart segmentation results. The projected segmentation methodology incorporates a mean field term inside the quality fuzzy c-means objective operate. Since mean shift will quickly and faithfully realize cluster centers, the whole strategy is capable of effectively police investigation regions inside a picture.
\end{abstract}

Keywords: computer vision, dermoscopy, melanoma, pattern analysis, skin cancer. Dermoscopy, fuzzy c-means, image segmentation, mean shift, melanoma, carcinoma..

\section{INTRODUCTION}

Skin cancer is now a days one among the leading cancers like totally different skin cancers, are alone really rarely among many white-skinned populations round the world irritating to the bit. inside the bulk of cases theylll're [1]. modification of recreational behavior along side the detected alone by their look. it's vital to urge eliminate a increase in ultraviolet cause a dramatic increase inside the melanoma early in its life. usually|this can be $\}$ often as a range of melanomas diagnosed. The raise in incidence was results of "thin" melanoma have a sensible prognosis (96\% firstly observed in the u.s. in 1930, wherever one person cure rates). thus early detection is very important in out of 1 hundred 000 once a year suffered from cancer. dominant the unwellness. the foremost common web site This rate increased inside the center of the eighties to six for melanoma in men is that the rear and in girls the legs. per 100000 [2] and to 13 per 100000 in 1991. The However, you got to check all of our skin.

numbers area unit love the incidence rates discovered in Europe. In 1995, in Republic of European nation the incidence of melanoma was relating to twelve per 100 000 , that reflected an increase of $51.8 \%$ inside the previous ten years [3], and so the incidence of melanoma shows a still increasing tendency. melanoma and NonMelanoma these are two main varieties of skin cancers. each style of skin cancer is totally totally different from the alternative carcinomas in sure characteristics melanoma is that the foremost dangerous of the common varieties of cancer.

Melanoma generally looks as degree enlarging the colored skin spot. they will have varied reminder brown, black and blue. they will be flat or raised. a little proportion of melanoma don't appear to be vivacious, but appear as a high-powered of intensity as a "colored skin" spot.Keep in mind that the majority of melanoma donIII't have any symptoms when they are found. Some is additionally fretful, and injury could also be a late sign. Melanomas,

The most necessary common risk issue for developing skin cancer has several moles. alternative necessary risk factors embody a case history of skin cancer, a private history of skin cancer or alternative varieties of carcinoma, lentiginous or truthful skin (skin that tends to burn instead of $\tan$ ), and frequent recreational sun exposure. whereas skin \{cancer\} can occur in childhood itl's uncommon before time of life.

Our skin protects our body from heat, injury, and infection. It conjointly protects our body from harm caused by ultraviolet (UV) radiation (such as from the sun or sunlamps) [22]. Our skin stores water and fat. It helps management body heat. Also, your skin makes cholecarciferol.

The Figure one. 1 on subsequent page shows the 2 main layers of the skin The Figure 1.1 on the next page shows the two main layers of the skin. 

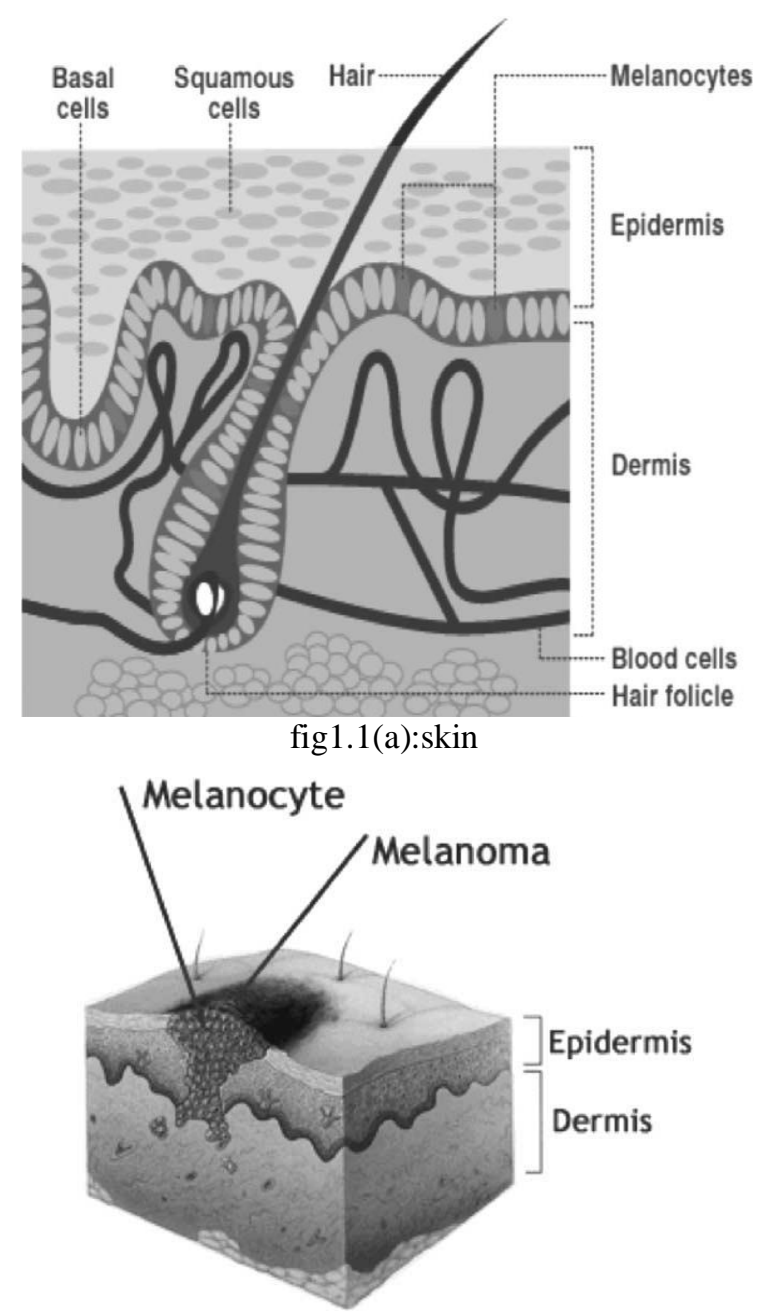

fig (b):infected skin

Figure 1.1 (a) skin (b) infected skin [13]

\section{LITERATURE REVIEW}

In this chapter we are going to target work done by numerous folks on carcinoma detection by mistreatment totally different techniques like FCM, K-means, GLCM, Contour Signature and SVM.

According to the importance of early detection, many corporations have dedicated time and efforts to boost the first screening method. one in all the foremost effective solutions already developed was the results of Australian Commonwealth Scientific and Industrial analysis Organization analysis.

It captures and analyses label pictures of pigmented skin lesions that square measure hold on for subsequent lesion observation or confirmation. The system was tested and one paper revealed within the application of the system. In the year of 2002 Thomas Bayes rule in concert of the skin lesions classification technique was revealed with rather inconclusive results. Common classification strategies like applied mathematics and rule-based ones were applied within the researches of. $\mathrm{K}$ nearest neighborhood as another classification technique was used within the analysis .

\section{A] Preprocessing Techniqes:}

Images square measure typically corrupted by random variations in intensity values, known as noise. Some common forms of noise square measure salt and pepper noise, impulsenoise, and mathematician noise. Salt and pepper noise contains random occurrences of each black and white intensity values. However, impulse noise contains solely random occurrences of white intensity values. not like these, mathematician noise contains variations in intensity that square measure drawn from a mathematician or statistical distribution and could be a excellent model for several forms of sensing element noise, like the noise as a result of camera natural philosophy. Linear smoothing filters square measure sensible filters for removing mathematician noise and, in most cases, the opposite forms of noise still. A linear filter is enforced mistreatment the weighted total of the pixels in serial windows.In image preprocessing there square measure thus mane filters used for pre method like linear filters and nonlinear filters. one in all the best linear filters is enforced by native|an area|a neighborhood \} averaging operation wherever the worth of every picture element is replaced by the typical of all the values within the local neighborhood. Its known as mean fiter. mathematician filters square measure a category of linear smoothing filters with the weights chosen per the form of a mathematician operate. The mathematician smoothing filter could be a excellent filter for removing noise drawn from a standard distribution.

B] Segmentation Techniques:

The division of a picture into significant structures, image segmentation, is commonly anessential step in image analysis, object illustration, mental image, and manyother image process .A great form of segmentation ways has been planned within the past decades, and a few categorization is critical to gift the ways properly here. A disjunct categorization doesnl't appear to be potential thol', as a result of even 2 terribly completely different segmentation approaches might share properties that defy singular categorization. Threshold based mostly segmentation. bar chart thresholding and slicing techniquesare accustomed section the image. they will be applied on to a picture, however canalso be combined with pre- and post-processing techniques.Edge based mostly segmentation-With this method, detected edges in a picture areassumed to represent object boundaries, and accustomed establish these objects.Region \{based|based mostly|primarily based mostly\} segmentation-Where a foothold based technique might arrange to realizethe object boundaries and so find the thing itself by filling them in, a regionbased technique takes the alternative approach, by (e.g.) beginning within the middle of anobject and so "growing" outward till it meets the thing boundaries.Clustering techniques-Although clump is usually used as a equivalent word for(agglomerative) segmentation techniques, we tend to use it here to denote techniques thatare primarily employed in explorative information analysis of high-dimensional measurementpatterns. during this context, clump ways 
arrange to cluster along patternsthat square measure similar in some sense. This goal is extremely almost like what we tend to square measure attemptingto do after we section a picture, and so some clump techniques canreadily be applied for image segmentation.MatchingWhen we all know what associate degree object we tend to would like to spot in a picture (approximately)looks like, we are able to use this information to find the thing in a picture. This approach to segmentation is termed matching.

\section{Thresholding based mostly Segmentation:}

Thresholding is maybe the foremost often used technique to section a picture. The thresholding operation may be a gray price remapping operation. Thresholding maps agreyvalued image to a binary image. once the thresholding operation, the image hasbeen divided into 2 segments, known by the pel values zero and one severally.If wel've a picture that contains bright objects on a dark background, thresholdingcan be accustomed section the image. Since in manytypes of pictures the gray prices of objects square measure terribly completely different from the background value,thresholding is commonly a well-suited technique to section a picture into objects and background.If the objects arenl't overlapping, then we are able to produce a separate section fromeach object by running a labelling formula on the thresholded binaryimage, therefore distribution a novel pel price to every object. several ways exist to pick an appropriate threshold price for a segmentation task. Perhapsthe commonest technique is to line the edge price interactively; the user manipulatingthe price and reviewing the thresholding result till a satisfying segmentation hasbeen obtained. The bar chart is commonly a valuable tool in establishing an appropriate thresholdvalue. several ways exist to seek out an appropriate threshold for segmentation. the best technique is that the interactive choice of a threshold by the user -possibly with the help of the image histogram - a way thatl's typically in the midst of a graphical tool that lets the user like a shot assess the results of a definite selection of threshold. Automatic ways typically create use of the image bar chart to seek out an appropriate threshold.

\section{C] Texture Classification:}

The textures square measure described by texels. The identification of specific textures in a picture is finished by modeling texture as a two-dimensional (2D) grey level variation. By estimating degree of distinction, regularity, coarseness and radial asymmetry, the relative brightness of pixels square measure computed.

\section{Color pattern Classification:}

It is supported the colour matching within the divided image. The computation of a color histogram from divided image is that the most significant step to spot the proportions of pixels at intervals a picture that holds specific values that we tend to humans specific as colours. Color examination doesn l't depend upon image size or its orientation and color classification usually involves comparison of color histograms.
D] Feature Analysis:

Image analysis techniques involves the activity of extracted image options. activity of image options for identification of skin cancer needs that initial, the lesions be detected and localized in a picture. It's essential that lesion boundaries square measure determined accurately in order that measurements, e.g. most diameter, asymmetry, irregularity of the boundary, and color characteristics is accurately computed. Textural Analysis is that the arrange to quantify texture notions like "fine," "rough" and "irregular" and establish, live and utilize the variations between them. Textural options and textural analysis ways is loosely divided into 2 classes, viz., applied math and structural. applied math ways outline texture in terms of native gray-level statistics that square measure constant or slowly varied over a rough-textured region. \{different|totally completely different|completely different textures is discriminated by comparison the statistics computed over different sub-regions. a number of the foremost common textural options square measure, neighboring gray-level dependence matrix, unsimilarity, angular moment, GLCM variance.

\section{III . BLOCK DIAGRAM}

Methodology block is as given in below figure:

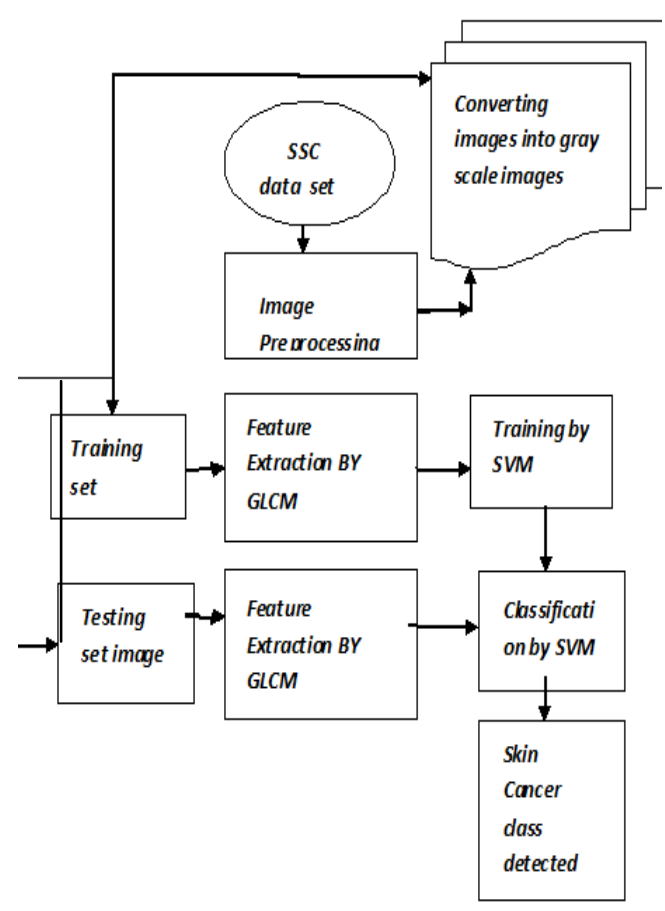

Block diagram[27]

A] Pre-Processing Of Image:

Most pictures square measure affected to some extent by noise, that l's unexplained variation in data:

disturbances in image intensity that square measure either uninterpretable or not of interest. Image analysis is usually simplified if this noise will be filtered out. In an identical method filters square measure utilized in chemistry to free liquids from suspended impurities by passing them through a layer of sand or charcoal. Engineers operating in signal process have extended the which means of the term 
filter to incorporate operations that intensify options of interest in information. using this broader definition, image filters could also be wont to stress edges -that is, boundaries between objects or elements of objects in pictures. Filters give associate degree aid to visual interpretation of pictures, and might even be used as a precursor to more digital process, like segmentation.

When a picture is noninheritable by a camera or alternative imaging system, usually the vision system that itl's supposed is unable to use it directly. The image could also be corrupted by random variations in intensity, variations in illumination, or poor distinction that has to be restrained within the early stages of vision process. This section discusses ways for image preprocessing aimed toward eliminating these undesirable characteristics. the most downside with native averaging operations is that they have an inclination to blur sharp discontinuities in intensity values in a picture. an alternate approach is to switch every component worth with the median of the grey values within the native neighborhood. Filters victimization this method square measure referred to as median filters [2] [15]. Median filters square measure terribly effective in removing salt and pepper and impulse noise whereas retentive image details as a result of they do|they square measure doing $\}$ not rely upon values that are considerably totally different from typical values within the neighborhood. Median filters add consecutive image windows in a very fashion like linear filters. However, the method isnl't any longer a weighted add. for instance, take a three $\mathrm{x}$ three window and work out the median of the pixels in every window targeted around .

1. kind the pixels into ascending order by grey level.

2. choose $\{$ the worth|the worth of the center component because the new value for component.

In general, associate degree odd-size neighborhood is employed for calculative the median. However, if the amount of pixels is even, the median is taken because the average of the center 2 pixels when sorting.

Noise arises in several stages of image. Preprocessing is to perform image process on original image by victimization median filter to get increased image. The median filter preserve the amplitude and site of edges. The median filter reduces the variance of the intensities within the image. A median filter smoothers the image by utilizing the median of the neighborhood. The construct of a median filter was introduced by Tukey in 1977. Its extension to two-dimensional pictures was mentioned by Pratt in 1978. The median filter preserves the amplitude and site of edges and steps higher than smoothing, whereas reducing the noise. Image smoothing is that the set of native pre-processing ways that have the aim of suppressing image noise - it uses redundancy within the image information. Calculation of the new worth is predicated on averaging of brightness values in some neighborhood. Smoothing poses the matter of blurring sharp edges within the image, and then we have a tendency to shall think about smoothing ways that square measure edge conserving. theyl're supported the overall concept that the typical is computed solely from those purposes within the neighborhood that have similar properties to the processed point.

Local image smoothing will effectively eliminate impulsive noise or degradations showing as skinny stripes, however doesnl't work if degradations square measure massive blobs or thick stripes. when preprocessing of input image wel'll move towards the segmentation.

\section{B] Segmentation:}

Various techniques will be use to Segmentation of pictures. Here wel'll use Fuzzy C-Means (FCM) for the segmentation type carcinoma image[4] [6] [12] [15] [16]. Image segmentation is that the division of a picture into regions or classes, that correspond to totally different objects or elements of objects. each component in a picture is allotted to 1 of variety of those classes. an honest segmentation is usually one in which: pixels within the same class have similar gray scale of variable values and type a connected region, neighboring pixels that square measure in numerous classes have dissimilar values. Segmentation is usually the crucial step in image analysis: the purpose at that we have a tendency to move from considering every component as a unit of observation to operating with objects (or elements of objects) within the image, composed of the many pixels. If segmentation is completed well then all alternative stages in image analysis square measure created less complicated. There square measure 3 general approaches to segmentation, termed thresholding, edge-based ways and region-based ways.

In thresholding, components square measure allotted to classes in keeping with the vary of values inwhich a pixel lies [9] [11].The boundaries between adjacent pixels in numerous classes has been superimposed in white on the first image. It will be seen that the edge has with success segmental the image into the 2 predominant fibre sorts.

In edge-based segmentation, a grip filter is applied to the image, pixels square measure classified as edge or nonedge counting on the filter output, and pixels that arenl't separated by a grip square measure allotted to constant class [9]. Finally, region-based segmentation algorithms operate iteratively by grouping along pixels that square measure neighbours and have similar worths and rending teams of pixels that square measure dissimilar in value [6]. Segmentation is one in all the necessary steps in cancer detection. The aim of segmentation method is to divide the image into homogenized, consistent regions, that ought to correspond to totally different objects within the scene. the method is achived victimization solely properties of image. The properties square measure edges and texture square measure helpful for segmentation. Image segmentation is that the method of partitioning a picture into teams of pixels that square measure undiversified with reference to some criterion. Segmentation cares with dividing a picture into purposeful regions.

\section{C] Fuzzy C-Means Segmentation:}

Fuzzy c-means (FCM) may be a information agglomeration technique whereby every information belongs to a cluster to a point thatl's fixed by a 
membership grade [4] [6] [14] [15] [16] [17]. this method was originally introduced by Jim Bezdek in 1981 [Bez81] as associate degree improvement on earlier agglomeration ways. It provides a way that shows the way to cluster information points that populate some third-dimensional area into a particular variety of various clusters. The formal logic tool cabinet statement perform fcm starts with associate degree initial guess for the cluster centers, that square measure supposed to mark the mean location of every cluster.

The initial guess for these cluster centers is possibly incorrect. to boot, fcm assigns each information a membership grade for every cluster. By iteratively change the cluster centers and therefore the membership grades for every information, fcm iteratively moves the cluster centers to the \"rightl" location among a knowledge set. This iteration is predicated on minimizing associate degree objective perform that represents the gap from any given information to a cluster center weighted by that information pointl's membership grade. fcm may be a statement perform whose output may be a list of cluster centers and a number of other membership grades for every information. youl'll use the data came by fcm to assist you build a fuzzy abstract thought system by making membership functions to represent the fuzzy qualities of every cluster. Fuzzy C-Means is associate degree unattended agglomeration algorithmic program that has been applied to issues involving agglomeration feature analysis. FCM has additional applications like agricultural engineering, astronomy, geology, image analysis, diagnosing, form analysis, chemistry. Fuzzy C-Means segmentations is predicated on partial agglomeration. Fuzzy C-Means is additional versatile. within the carcinoma image, cancer is clearly seen by victimization median filter. Thus, we will divide segmentation image into 2 elements object and background half. The cluster region calculates 2 regions.

\section{D]Feature Analysis :}

At some purpose in image process a choice is formed regarding that image points or regions of the image square measure relevant for additional process. so as to tell apart regions in pictures itl's necessary to analyse their characteristics. Digital image characteristics square measure primarily three: color, form and texture [17] [18]. a decent descriptor for a picture, normally, integrates some data from all 3 options. color is said to human's perception and additionally to many medication specifications. the color of Associate in Nursing object in a picture depends each on physics and on perception, so itl's significantly complicated to characterise it. form refers to the define or the looks of one thing within the image that describes its geometrical properties. This characteristic will be accustomed fix patterns to classify objects with similar shapes, thence outline totally different teams of components with similar shapes. Texture might be understood because the look of a selected surface or a part of Associate in Nursing object in a picture.

In the same image totally different textures will be known process some regions. Combining this characteristic with some datum tools vital data will be extracted from the image. This assignment presents results and discussion a couple of set of computations enforced on many pictures. These computations were programmed to come up with texture descriptors exploitation co-occurrence matrices and energy filters. The results helped to work out the correctness of the input parameters for the co-occurrence matrix and therefore the energy filters looking on the feel sort. the most propose of the exercise is to implement basic principles on image texture analyses exploitation Matlab, and a lot of exactly co-occurrence matrices and energy filters combined with some statistics, like homogeneity, distinction and uniformity[18] [19] [20].

\section{E]Co-Occurrence Matrices:}

A co-occurrence matrix contains the frequency of a definite try of pixels repetition in a picture. so as to calculate a co-occurrence matrix itl's necessary to grasp the subsequent values:Number of gray levels: a grayscale image contains 256 grey levels, which suggests a high procedure price as a result of all potential component pairs should be taken in account. the answer is to come up with the matrix reducing the amount of gray scales, so the amount of potential component combos. The cooccurrence matrix is usually sq. with constant spatial property because the range of grey-levels chosen. This price is usually set to eight.Angle. equally to the space itl's necessary to outline the direction of the try of pixels. the foremost common directions square measure zero, $45^{\circ}$, $90^{\circ}, 135^{\circ}$, and its regular equivalents[19].

Contrast: Returns a live of the intensity distinction between a component and its neighbor over the full image.

$$
\sum_{i, j}|i-j|^{2} p(i, j)
$$

Range $=\left[0(\operatorname{size}(\mathrm{GLCM}, 1)-1)^{\wedge} 2\right]$

Contrast is zero for a continuing image.

Correlation: Returns a live of however related to a component is to its neighbor over the full image.

$$
\sum_{i, j} \frac{(i-\mu i)(j-\mu j) p(i, j)}{\sigma_{i} \sigma_{j}}
$$

Range $=\left[\begin{array}{ll}-1 & 1\end{array}\right]$

Correlation is one or -1 for a superbly absolutely or negatively related to image. Correlation is $\mathrm{NaN}$ for a continuing image.

Energy: Returns the add of square components within the GLCM.

$$
\sum_{i, j} p(i, j)^{2}
$$

Range $=\left[\begin{array}{ll}0 & 1\end{array}\right]$

Energy is one for a continuing image

Homogeneity: Returns a worth that measures the closeness of the distribution of components within the GLCM to the GLCM diagonal. 


$$
\sum_{i, j} \frac{p(i, j)}{1+|i-j|}
$$

Range $=\left[\begin{array}{ll}0 & 1\end{array}\right]$

Homogeneity is one for a diagonal GLCM

\section{F] Contour Signature :}

Form of image is nothing however distance of all the purpose on its boundary from some reference. Its additionally known as contour signature [5] [13]. This reference is center of mass of image. Center of circle is its center of mass and distances of all points from center square measure equal. For sq. itl'll vary. during this case wel've got thought-about points on the boundary of the image that square measure separated by angle of ten degrees. All the angle square measure measured from center of image. so wel've got calculated thirty six distance similar to thirty six totally different angles separated by ten degrees. This angle separation will be reduced so as to extend accuracy. however beside reduction in angle separation, range of reading can increase and it'lll increase computation time. therefore therel's tread off between ability of system to figure in real time and its accuracy.

\section{SKIN LESION CLASSIFICATION STRATEGIES}

In this section, the foremost in style strategies for skin lesion classificationare examined. The task involves primarily 2 phasesafter feature choice, learning and testing[22].

\section{A. Learning part:}

During the educational part, typical feature values square measure extracted from a sequence of digital pictures representing classified skin lesions. The support vector machine (SVM) may be a in style formula for data classification in 2 categories. SVMs allow the growth of the data provided by a learning dataset as a linear combination of a set of the information within the learning set (support vectors). These vectors find a hypersurface that separates the input file with a awfully sensible degree of generalization.

The SVM formula relies on learning, testing, and performance analysis, that square measure common steps in each learning procedure. Learning involves improvement of a convexo-concave cost perform wherever there arenl't any native minima to complicate the learning method. Testing relies on model analysis exploitation the support vectors to classify a take a look at dataset[23].

\section{B. Testing part:}

The performance of every classifier is tested exploitation Associate in Nursing ideally large set of manually classified pictures. A set of them, e.g., eightieth of the photographs, is used as a learning set, and therefore the alternative 2 hundredth of the samples is used for testing exploitation the trained classifier. the educational and take a look at pictures square measure changed for all potential combos to avoid bias within the answer[24-26].

\section{CONCLUSION}

Our planned system show that we have a tendency to reach improved classification results compared to the progressive international and moving ridge remodel primarily based descriptors. we have a tendency to conceive to extend our technique to find and classify the presence of alternative dermoscopic options (e.g. pigment network, dots, tube-shaped structure structures),moving U.S.A. a vital success towards a machine-learning primarily based laptop assisted diagnosing system for early detection of carcinoma. Our planned system is additionally capable of police investigation sorts of cancers by the employment of support vector machine.so that we are going to be able to find the cancer in early stages accurately.

\section{REFERENCES}

[1] Scott E.Umbaugh,Randy H.Moss, and William V.Stoecker,"Applying Artificial Intelligence to the Identification of Variegated Coloring in Skin Tumors",IEEE Engineering In Medicine And Biology,Magazine, Vol.10, No.4, pp 57-62, 1991.

[2] F.Ercal,M.Moganti,W.V.Stoecker, and R.H.Moss, "Detection Of Skin Tumor Boundaries in Color Images",IEEE Transactions On Medical Imaging, Vol.12,No.3, pp 624-626, September 1993".

[3] Fikret Ercal, Senior Member,IEEE Anurag Chawla,William V.Stoecker,Hsi-Chieh Lee, and Randy H.Moss,Senior Member,IEEE, "Neural Network Diagnosis of Malignant Melanoma From Color Images", IEEE Transactions On Biomedical Engineering,Vol.41,No.9, pp 837-845, September 1994.

[4] Philippe Schmid, "Segmentation of Digitized Dermatoscopic Images by Two-Dimensional Color Clustering", IEEE Transactions On Medical Imaging, Vol.18, No.2, pp 164-171, February 1999.

[5] Do Hyun Chung and Guillermo Sapiro,Member ,IEEE, "Segmenting Skin Lesions with Partial-Differential-Equations-Based Image Processing Algorithms", IEEE Transactions On Medical Imaging, Vol.19, No.7, pp 763-767, July 2000.

[6] Harald Ganster, Axel Pinz,Reinhard Rohrer,Ernst Wildling,Michael Binder,and Harald Kittler, "Automated Melanoma Recognition", IEEE Transactions On Medical Imaging, Vol.20, No.3, pp 233-239, March 2001.

[7] Tim K.Lee, M.Stella Aitkins,Michael A.King, Savio Lau, and David I.McLean, "Counting Moles Automatically From Back Images", IEEE Transactions On Medical Imaging, Vol.52, No.11, pp 19661969, November 2005.

[8] Xiaojing Yuan,Member IEEE,Zhenyu Yang,George Zouridakis,Senior Member IEEE, and Nizar Mullani,"SVM-based Texture Classification and Application to Early Melanoma detection", Proceedings of the 28th IEEE Embs Annual International Conference New York City, Usa, pp 4775-4778, Aug 30-Sept 3,2006.

[9] Jose Fernandez Alcon, Calina Ciuhu, Warner ten Kate,AdrienneHeinrich,Natallia Uzunbajakava,Gertruud Krekels, Denny Siem, and Gerard de Haan, "Automated Imaging System With Decision Support for Inspection of Pigmented Skin Lesions and Melanoma Diagnosis", IEEE Journal Of Selected Topics In Signal Processing, Vol.3, No.1, February 2009.

[10] B.Gracia Zapirain, A.Mendez Zorrilla ,I.Ruiz Oleagordia, G.Nunez,A.Abtane, "Skin Cancer Parameterisation Algorithm Based On Epiluminiscence Image Processing",IEEE International Sysposium on Signal Processing and Information Technology, pp 236-241, 2009

[11] Ho Tak Lau, Adel Al-Jumaily,"Automatically early Detection of Skin Cancer: Study Based on Nueral Netwok Classification",2009 International Conference of Soft Computing and pattern Recognition.pp 375-380, 2009.

[12] Margarida Silveria, Jacinto C. Nascimento, Jorge S. Marques, Andre R.S.Marcal, Teresa Mendonca, Syogo Yamauchi, Junji Maeda, and Jorge Rozeira, "Comparison of Segmentation Methods For Melanoma Diagnosis in Dermoscopy Images," IEEE Journal of Selected Topic in Signal Processing, vol.3, no.1, pp. 35-45, February 2009. 
[13] Ilias Maglogiannis, Member, IEEE, and Charalampos N. Doukas, Student Member, IEEE,’Overview of Advanced Computer Vision Systems for Skin Lesions Characterization," transactions on information technology in biomedicine, Vol 13,pp 721-733,2009.

[14] Huiyu Zhou, Gerald Schaefer, Abdul H. Sadka, and M.Emre, "Anisotropic Mean shift Based Fuzzy C-Means Segmentation of Dermoscopy Image," IEEE Journal of Selectd Topics in Signal Processing, vol.3. no. 1, pp 26-34, February 2009.

[15] Sookpotharom Supot, "Border Detection of Skin Lession Images Based on Fuzzy C-Means Thresholding," in Third International Conference on Genetic and Evolutionary Computing, pp. 777-780, 2009

[16] Shang Keke, Zhou Peng, and Li Guohui, "Study on Skin Color Image Segmentation used by Fuzzy C-Means Arithmetic," in Seventh International Conference on Fuzzy Systems and Knowledge Discovery (FSKD 2010).vol. 2, pp. 612-615,2010.

[17] Azaden Noori Hoshyar, Adel Al-Jumaily, and Riza Sulaiman,"Review On Automatic Early skin Cancer Detection," in International Conference in Computer science and Service System(CSSS), pp.4036-4039, 2011.

[18] Lucia Ballerini , Robert B. Fisher ,Ben Aldridge, and Jonathan Rees, "Non- Melanoma Skin Lesion Classification Using Colour Image Data In A Hierarchical K-NN Classifier", IEEE Conference in Biomedical Imaging, pp 358-361, 2012.

[19] R. Subash Chandra Boss, K. Thangavel, and D. Arul Pon Daniel,"Mammogram Image segmentation Using Fuzzy Clustering" IEEE Conference on Pattern Recognition, Informatics and Medical engineering, pp no 290-295, 2012.

[20] J.Abdul Jaleel, Sibi Salim, and Aswin.R.B, “ Computer Aided Detection of Skin Cancer," IEEE Conference on Circuits, Power and $J$ Kingravi, B. Uddin, H. Iyatomi, Y. A. Aslandogan, W. V. Stoecker, and R. H. Moss, "A methodological approach to the classification of dermoscopy images," Comput. Med. Imag. Graph.,vol. 31, pp. 362-373, Mar. 2007.

[24] C. Burges. (2001). A tutorial on support vector machines for pattern recognition [Online]. Available: http://www.kernel-machines.org/

[25] N. Christianini and J. Shawe-Taylor, An Introduction to Support Vector Machines. Cambridge, U.K.: Cambridge Univ. Press, 2000.

[26] B. Sch“olkopf. (2000). Statistical learning and kernel methods [Online]. Available:http://research.Microsoft.com/ bsc

[27] Ritesh Maurya,Surya Kant Singh,Ashish k Maurya,"GLCM and Multiclass Support Vector Machine based Automated Skin Cancer Classification",IEEE 2014 